\title{
Revulsion in chimpanzees: health maintenance through avoidance of biological contaminants
}

\section{AUTHOR(S):}

Sarabian, Cecile; Maclntosh, Andrew J. J.

\section{CITATION:}

Sarabian, Cecile ...[et al]. Revulsion in chimpanzees: health maintenance through avoidance of biological contaminants. PeerJ Preprints 2016, 4: e1851v1.

\section{ISSUE DATE:}

2016-03-11

URL:

http://hdl.handle.net/2433/232530

\section{RIGHT:}

(c) 2016 Sarabian et al.; This is an open access article distributed under the terms of the Creative Commons Attribution License, which permits unrestricted use, distribution, reproduction and adaptation in any medium and for any purpose provided that it is properly attributed. For attribution, the original author(s), title, publication source (PeerJ Preprints) and either DOI or URL of the article must be cited. 


\title{
Revulsion in chimpanzees: health maintenance through avoidance of biological contaminants
}

\author{
Cecile Sarabian ${ }^{1}$ and Andrew J. J. Maclntosh ${ }^{1,2}$ \\ 1. Primate Research Institute, Kyoto University, Inuyama, Japan \\ 2. Wildlife Research Center, Kyoto University, Sakyo, Japan
}

Presenter's email: sarabiancecile@gmail.com

Keywords: Parasite Avoidance Theory of Disgust, bodily products, health, genus Pan

Many major human infectious diseases are transmitted via bodily products such as feces, blood, semen, and saliva. Such contaminants are also known disgust elicitors among humans. From an evolutionary perspective, revulsion in humans is hypothesized to have evolved to protect us from the risk of contracting disease; sensu the Parasite Avoidance Theory of Disgust. To investigate whether chimpanzees are sensitive to these potential sources of infection, we conducted feeding-related experiments to elicit avoidance behaviors in captive chimpanzees (Pan troglodytes troglodytes, $\mathrm{N}=20-42$; sex ratio $=1 ; 27 \pm 9$ years-old) at the Centre International de Recherches Médicales de Franceville, Gabon. In the first set of experiments, we tested for fecesavoidance behaviors by presenting target individuals with food rewards in conjunction with visual, olfactory and tactile cues of chimpanzee feces. In a second experiment, the same individuals were presented with olfactory stimuli of blood, semen, and rotten meat. In all experiments, chimpanzees expressed some degree of behavioral avoidance, manifest as refusals to feed, increased latencies to feeding, or maintenance of greater distances from contaminants relative to control substrates. We are now in the process of testing whether behavioral tendencies observed toward these contaminants coincide with individual health markers, including current levels as well as historical records of infection with bacterial, protozoan and helminth parasites. We predict that individuals with greater tendencies toward avoidance of such contaminants, possibly representative of revulsion, to be characterized by better general health than less sensitive individuals. 